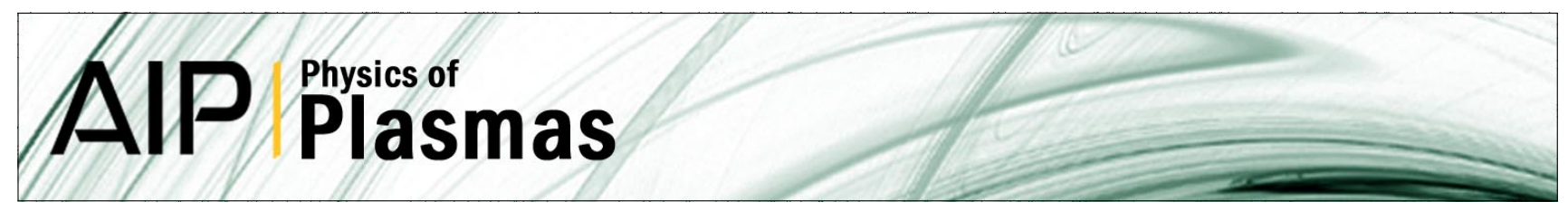

\title{
Ion holes in the hydrodynamic regime in ultracold neutral plasmas
}

P. McQuillen, J. Castro, T. Strickler, S. J. Bradshaw, and T. C. Killian

Citation: Phys. Plasmas 20, 043516 (2013); doi: 10.1063/1.4802813

View online: http://dx.doi.org/10.1063/1.4802813

View Table of Contents: http://pop.aip.org/resource/1/PHPAEN/v20/i4

Published by the American Institute of Physics.

\section{Additional information on Phys. Plasmas}

Journal Homepage: http://pop.aip.org/

Journal Information: http://pop.aip.org/about/about_the_journal

Top downloads: http://pop.aip.org/features/most_downloaded

Information for Authors: http://pop.aip.org/authors

\section{ADVERTISEMENT}

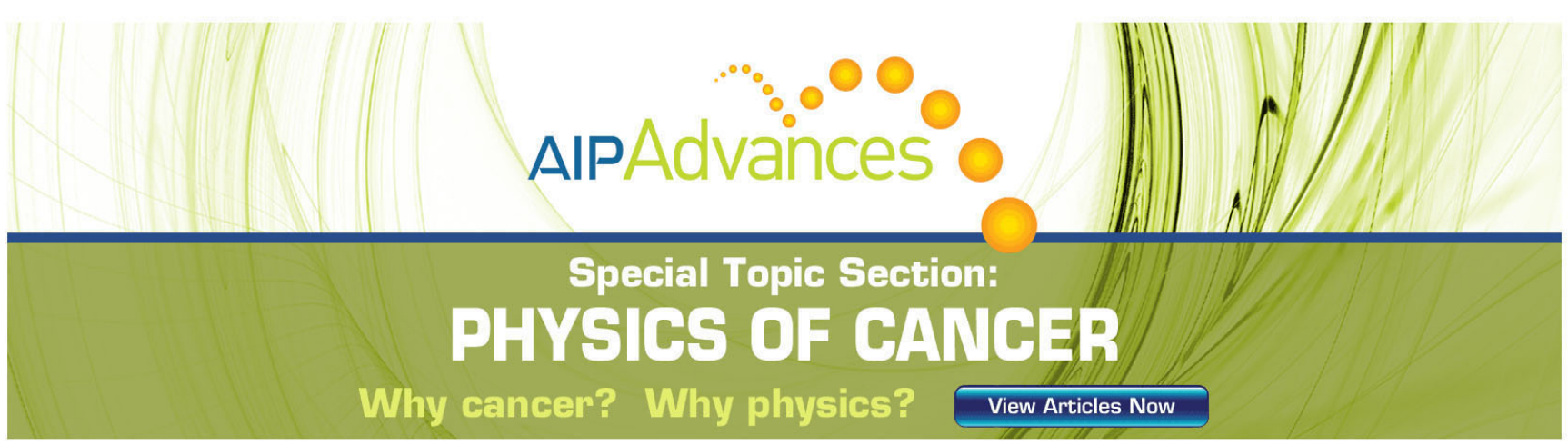




\title{
Ion holes in the hydrodynamic regime in ultracold neutral plasmas
}

\author{
P. McQuillen, ${ }^{\text {a) }}$ J. Castro, T. Strickler, S. J. Bradshaw, and T. C. Killian \\ Department of Physics and Astronomy, Rice University, Houston, Texas 77005, USA
}

(Received 1 April 2013; accepted 8 April 2013; published online 26 April 2013)

\begin{abstract}
We describe the creation of localized density perturbations, or ion holes, in an ultracold neutral plasma in the hydrodynamic regime, and show that the holes propagate at the local ion acoustic wave speed. We also observe the process of hole splitting, which results from the formation of a density depletion initially at rest in the plasma. One-dimensional, two-fluid hydrodynamic simulations describe the results well. Measurements of the ion velocity distribution also show the effects of the ion hole and confirm the hydrodynamic conditions in the plasma. (C) 2013 AIP Publishing LLC. [http://dx.doi.org/10.1063/1.4802813]
\end{abstract}

\section{INTRODUCTION}

Ultracold neutral plasmas (UNPs), ${ }^{1,2}$ formed through near-threshold photoionization of laser cooled atoms, occupy a unique parameter regime of neutral plasmas and also offer powerful diagnostics and excellent control over initial conditions. This makes them an excellent platform for studying a wide range of plasma phenomena, such as equilibration of strongly coupled plasmas, ${ }^{3-14}$ ambipolar diffusion with ${ }^{15}$ and without ${ }^{16,17}$ a magnetic field, electron plasma oscillations, ${ }^{16,18}$ Tonks-Dattner resonances ${ }^{19}$ and edge modes, ${ }^{20}$ ion acoustic waves, ${ }^{21}$ an electron drift instability, ${ }^{22}$ threebody recombination at ultracold temperatures, ${ }^{13,23-29}$ and the crossover to an ultracold plasma from a dense gas of Rydberg atoms. ${ }^{30-33}$ Recent experiments creating ultracold plasmas in a seeded supersonic molecular beam ${ }^{34}$ introduce molecular processes to the plasma evolution and show promise for yielding more strongly coupled systems. Here, we take advantage of the ability to control the initial density distribution to create and study localized density perturbations, or ion holes, in an UNP in the hydrodynamic regime.

Propagating localized density perturbations in a neutral plasma are of great interest because they take many forms and can be sensitive to nonlinear and kinetic effects. ${ }^{35,36}$ Local density enhancements in a plasma are typically referred to as solitons ${ }^{37,38}$ and depletions are referred to as holes. ${ }^{35-37}$ Here, we focus on density depletions in which ion and electron densities closely follow each other, which are referred to as ion holes. ${ }^{39,40}$ We create ion holes by sculpting the initial density distributions to have a planar depletion through the center of the plasma. We find that the hole spontaneously splits into two planar holes that propagate at the local ion acoustic speed. This behavior is further characterized by simulating the plasma evolution with a onedimensional two-fluid simulation that was originally developed to investigate solar and stellar atmospheres. ${ }^{41,42}$

For the conditions of our experiment, the ion holes are well described as linear ion acoustic phenomena in a hydrodynamic regime, similar to ion acoustic waves previously studied in UNPs. ${ }^{21}$ Similar ion holes are also seen in non-

\footnotetext{
a)patrickmcquillen@rice.edu
}

neutral plasmas ${ }^{37}$ and dusty plasmas in the form of dust-ionacoustic holes. ${ }^{43}$ Gap-splitting and hole-propagation are readily observed in hydrodynamic environments in general, even in relatively exotic systems such as quantum degenerate bosonic $^{44}$ and fermionic ${ }^{45}$ superfluids in ultracold atomic gases.

While the results presented here do not access a regime in which kinetic effects appear to be important, such experiments are possible in UNPs. So, it is worth noting that there is a rich literature on ion holes in the kinetic regime, which are important in many space plasma settings and experimental plasmas ${ }^{36,46,47}$ such as beam plasma heating. ${ }^{39}$ They are associated with two-stream instabilities, ${ }^{39,40}$ and the resulting negative potential produces a population of trapped ions that can create nonlinear effects even in the small-amplitude limit. ${ }^{35,36}$ The interactions of overlapping colliding density perturbations have also attracted significant interest because of their possible role in the creation of energetic particles and other collective excitations. ${ }^{35,47}$ Electron holes are also possible in the electron component of a neutral plasma. ${ }^{48,49}$

\section{EXPERIMENTAL SETUP}

Ultracold neutral plasmas are created through photoionization of laser-cooled strontium atoms from a magnetooptical trap (MOT) as described in Ref. 3. The plasma density distribution is a spherical Gaussian, $n(r)=n_{0} \exp \left(-r^{2} / 2 \sigma_{0}^{2}\right)$, with $n_{0}=1.2 \times 10^{15} \mathrm{~m}^{-3}$ and $\sigma_{0}=1.4 \mathrm{~mm}$. The initial electron temperature is tunable $\left(T_{e}(0) \approx 1-1000 \mathrm{~K}\right)$, and is determined by the excess energy of the ionizing photons above threshold. Ion temperatures are set by disorder-induced heating ${ }^{4,7}$ to a density-dependent $T_{i}(0) \propto n^{1 / 3}$, which is approximately $1.5 \mathrm{~K}$ for our conditions. This results in strongly coupled ions in the liquid-like regime, ${ }^{2,50}$ but strong coupling does not have a significant effect on the observations described here.

To create ion holes, a planar density depletion cutting through the center of the plasma is formed by spatial modulation of the ionizing beam's intensity profile. To create small amplitude perturbations (10\% depletion), intensity masks are made by printing a $2 \mathrm{pt}$. (700 $\mu$ m wide) line on a transparency with a conventional inkjet printer. The mask is imaged onto the laser-cooled atoms with a 1:1 optical relay 
that provides excellent optical performance over the depth of field of the atom cloud down to $\sim 20 \mu \mathrm{m}$ length scales. Similar techniques were recently used to create a periodic density modulation and excite ion acoustic waves. ${ }^{21}$

Doppler-sensitive, laser-induced fluorescence (LIF) is used as a diagnostic. A tunable, narrowband laser induces fluorescence from the primary $\mathrm{Sr}^{+}$transition, ${ }^{2} \mathrm{~S}_{1 / 2}-{ }^{2} \mathrm{P}_{1 / 2}$ at $\lambda=422 \mathrm{~nm} .{ }^{17}$ The $422 \mathrm{~nm}$ laser beam illuminates the entire plasma and is close to perpendicular to the ionization beam and plane of the density depletion. Fluorescence emitted close to perpendicular to the plane of the ionizing and $422 \mathrm{~nm}$ beams is imaged through a 1:1 optical relay onto an intensified CCD camera. The point spread function of the imaging system is measured experimentally to have a FWHM of $25 \mu \mathrm{m}$, and is used to deconvolve acquired images to minimize spatial blurring. ${ }^{51}$ The resulting image, $F(\nu, x, y)$ depends on the laser frequency $(\nu)$ due to the natural linewidth and Doppler-broadening of the transition. ${ }^{21,52}$

Images are combined to obtain a map of the areal density, from which we infer the density distribution of the ions in the central plane, perpendicular to the gap $n_{i}(x, y, z \approx 0)$ $\propto \sum_{\nu} F(\nu, x, y)$, as described in Ref. 21. To probe the ion velocities and local temperature during the evolution of the ion hole, we examine LIF spectra of small spatial regions in the vicinity of the gap. ${ }^{21,52} \mathrm{We}$ obtain the spectrum of a region of the plasma, $S(\nu)_{\text {region }}$, by summing the fluorescence from that region

$$
S(\nu)_{\text {region }} \propto \sum_{x, y \in \text { region }} F(x, y, \nu) .
$$

Spectra can be fit by Voigt profiles convolving a Lorentzian function describing the natural linewidth of the transition and a shifted Gaussian for Doppler effects. The shift of the Gaussian represents the mean velocity of the ion population and the width represents the RMS velocity deviation (typically expressed as an effective ion temperature). ${ }^{52}$ To good approximation, because the Doppler-broadening is large, the spectrum can be interpreted as a distribution function for ion velocity along the laser beam (v) through the relation $\mathrm{v}=\lambda \delta \nu$, where $\delta \nu$ is the frequency offset from resonance.

We also model the plasma evolution with a onedimensional two-fluid simulation ${ }^{41,42}$ in which we account for bulk flows (transport, compression, and rarefaction), energy exchange between particle species by collisions and the work done by small-scale electric fields. We found that thermal conduction and viscous interactions made negligible contributions to the plasma energy balance in the temperature regimes explored here. The initial density distribution is matched to the profile observed experimentally within $100 \mathrm{~ns}$ of plasma creation. Ion temperature is set equal to the value after disorder-induced heating ${ }^{4}$ measured with LIF spectroscopy. ${ }^{52} \mathrm{~A}$ one-dimensional simulation will not correctly reproduce the adiabatic cooling of electrons and ions caused by the three dimensional expansion of the ultracold plasma. So in the simulation, we set the electron temperature using the known functional form for a self-similar expansion, ${ }^{17}$ which is determined by the initial electron temperature and size of the plasma (details given in Sec. III B). The ion hole propagation is insensitive to the ion temperature for our conditions of $T_{i} \ll T_{e}$.

\section{RESULTS AND DISCUSSION}

Figure 1 shows the evolution of the ion density distribution for a large amplitude perturbation. Immediately after plasma formation, a central gap is evident (Fig. 1(a)) with a length scale for the sides of the gap of about $\ell=200 \mu \mathrm{m}$. Ions move into the gap and a density feature emerges at the confluence. This evolves into two distinct density depletions, or ion holes, that propagate away from the plasma center. In spite of the inhomogeneous background density of the plasma, the propagation fronts remain close to parallel because the sound-speed depends only on the mass of the strontium ions and electron temperature, which is uniform across the front.

Figures 2(a) and 2(b) show density traces along a line through the center of the plasma, perpendicular to the initial density depletion, for two initial electron temperatures. For both conditions, we see an overall expansion of the plasma density profile with time, which has been well studied. ${ }^{17} \mathrm{We}$ also see an initial filling of the gap, appearance of a central density peak, and eventual splitting of the gap into two propagating ion holes. For the different electron temperatures shown, notice the similarity in the evolution but the difference in timescales, which scale with $T_{e}(0)^{-1 / 2}$. The central peak in density eventually matches the amplitude of the unperturbed cloud as seen in gap-splitting studies in hydrodynamic quantum fluids. ${ }^{44,45}$ The one-dimensional simulation accurately reproduces the evolution of the experimentally observed density profile.

\section{A. Hydrodynamic criteria}

The hydrodynamic nature of the plasma is important for this phenomenon. The critical parameter which demarcates hydrodynamic (collisional) and kinetic (collisionless) regimes is the ratio between the mean-free-paths of the particles and a characteristic scale-length of the plasma, known as the Knudsen number. When this ratio is small, the plasma is collisional and the particle velocity distribution functions are near Maxwellian. When the mean-free-path exceeds the characteristic scale-length then non-local phenomena can become important.

Tables I and II show the mean-free-paths and collision timescales for ion-ion and electron-electron collisions, respectively, for conditions relevant to strontium ultracold plasmas. They are defined in terms of the generalized collision frequency for a test particle $(\alpha)$ streaming through a background of field particles $(\beta)$

$$
\nu^{\alpha, \beta}=\left(1+m_{\alpha} / m_{\beta}\right) \psi\left(x^{\alpha, \beta}\right) \nu_{0}^{\alpha, \beta},
$$

where

$$
\begin{gathered}
\psi(x)=\frac{2}{\sqrt{\pi}} \int_{0}^{x} t^{1 / 2} \exp (-t) d t \\
x^{\alpha, \beta}=\frac{m_{\beta} v_{\alpha}^{2}}{2 k_{B} T_{\beta}},
\end{gathered}
$$



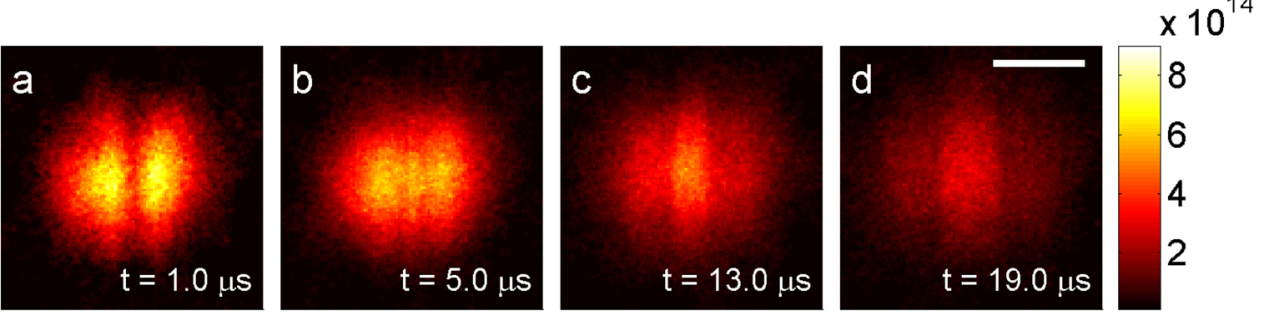

FIG. 1. False color plots of the ion density distribution for initial $T_{e}=25 \mathrm{~K}$.

The time since ionization is indicated. The initial localized density depletion splits to produce two ion holes propagating away from plasma center. (Scale $\mathrm{bar}=2 \mathrm{~mm}$, density given in $\mathrm{m}^{-3}$.)

and

$$
\nu_{0}^{\alpha, \beta}=\frac{4 \pi e_{\alpha}^{2} e_{\beta}^{2} \lambda_{\alpha, \beta} n_{\beta}}{m_{\alpha}^{2} v_{\alpha}^{3}} .
$$

$\lambda_{\alpha, \beta}$ is the Coulomb logarithm for the interaction. For ion-ion collisions of four times the thermal velocity $\left(v_{t h}\right)$ for a typical ion temperature of $T_{i}=1 \mathrm{~K}$, the mean-free-path is $73 \mu \mathrm{m}$, much less than the length scale of the gap in our experiments $(l=200 \mu \mathrm{m})$. At $4 v_{t h}$ when $T_{i}=2 \mathrm{~K}$, the mean-free-path is $300 \mu \mathrm{m}$, on the order of the length scale of the gap. So, kinetic effects due to the ions should be small in the parameter space of interest to us, which is supported by our observations. For collisions involving electrons, however, the meanfree-path approaches the gap dimension for one $v_{t h}$ at $T_{e}=$ $25 \mathrm{~K}$ and so electrons should behave kinetically, but these effects appear to be unimportant at the electron energies encountered here. They might become important in higher temperature plasmas where the streaming population could influence the energy balance and, consequently, the

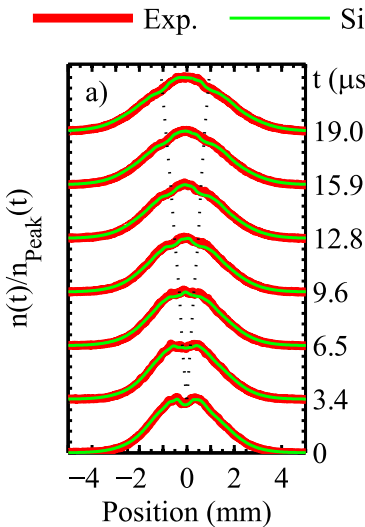

$$
\text { Sim. }
$$

Pos. Fits
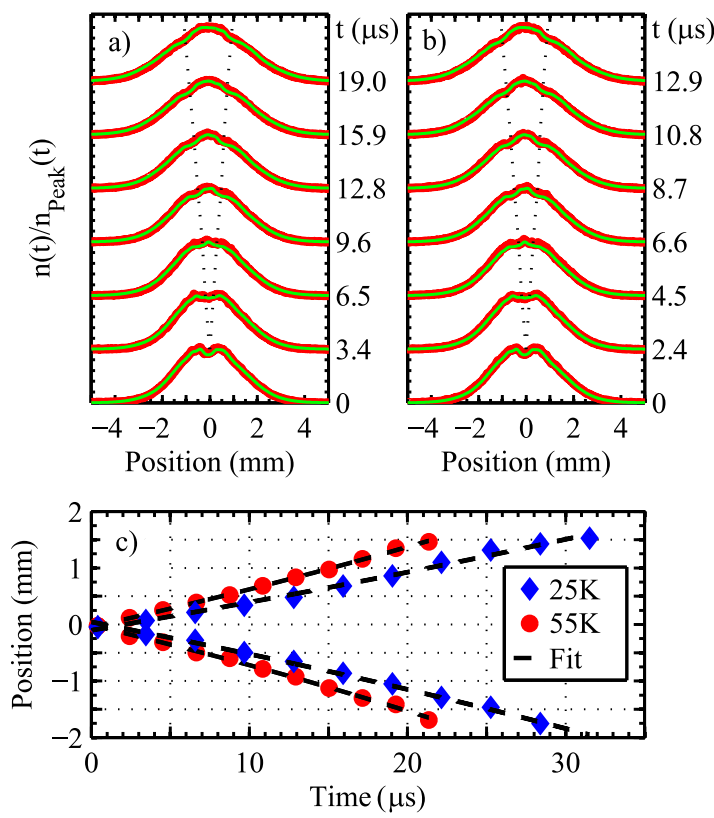

FIG. 2. Ion gap splitting and hole propagation for initial electron temperatures of $25 \mathrm{~K}$ (a) and $55 \mathrm{~K}$ (b). Data are slices along a line perpendicular to the initial density depletion through the center of the plasma, integrated over a width of $\pm \sigma(t) / 2$, with plasma size $\sigma(t)$ varying from $\sim 1.4$ to $2 \mathrm{~mm}$. The results of the hydrodynamic simulation and hole positions extracted from fits to the density profile are also shown. Traces are offset proportional to evolution time, $t$. (c) Positions of the holes with fits to Eq. (14). The average $T_{e}(0)$ values extracted from the hole propagation are $29 \pm 4 \mathrm{~K}$ and $48 \pm 4 \mathrm{~K}$. dynamics of the plasma via high order moments of the distribution function, such as the heat flux. However, within the parameter space explored here the behavior of the plasma is dominated by the ion dynamics, which are firmly within the collisional, hydrodynamic regime.

The hydrodynamic nature of the dynamics and the small amplitude of the density perturbations implies a simple explanation for the gap-splitting that is observed. The basic physics of the phenomenon should be described by the linear acoustic wave equation

$$
\frac{\partial^{2} n(x, t)}{\partial t^{2}}=C_{s} \frac{\partial^{2} n(x, t)}{\partial x^{2}} .
$$

Because the characteristic length scale of the perturbation is much larger than the electron Debye screening length, the ion acoustic wave-speed for any wave component significantly contributing to the ion-hole wave packet is constant, $C_{s}(t)={\sqrt{k_{B} T_{e}(t) / M}}^{53}$ Under these conditions, an initially stationary, localized perturbation, $n_{0}(x)$, will split into two traveling components described by $n(x, t)=\left[n_{0}\left(x+C_{s} t\right)\right.$ $\left.+n_{0}\left(x-C_{s} t\right)\right] / 2$.

By varying the properties of the induced plasma density distribution and the electron temperature, it is possible to look for kinetic effects on the plasma evolution. For example, we observe that with significantly higher electron temperature and smaller plasma density in the gap, corresponding to an ion mean-free-path much larger than the gap dimensions, the central feature is diminished or absent, and we see evidence for plasma streaming. In this paper, we focus on the hydrodynamic regime and leave these kinetic phenomena for a future study.

TABLE I. Mean-free-paths and timescales for Strontium ion-ion collisions, at a range of ion temperatures and multiples of the ion thermal speed, for a hole density of $10^{9} \mathrm{~cm}^{-3}$.

\begin{tabular}{lccc}
\hline \hline$T_{i}=$ & $1 \mathrm{~K}$ & $2 \mathrm{~K}$ & $3 \mathrm{~K}$ \\
\hline & & Mean-free-paths $(\mathrm{cm})$ & \\
$\frac{1}{2} v_{t h}$ & $2.20 \times 10^{-5}$ & $8.80 \times 10^{-5}$ & $1.98 \times 10^{-4}$ \\
$v_{t h}$ & $6.65 \times 10^{-5}$ & $2.67 \times 10^{-4}$ & $6.00 \times 10^{-4}$ \\
$2 v_{t h}$ & $4.79 \times 10^{-4}$ & $1.92 \times 10^{-3}$ & $4.31 \times 10^{-3}$ \\
$4 v_{t h}$ & $7.30 \times 10^{-3}$ & $2.92 \times 10^{-2}$ & $6.55 \times 10^{-2}$ \\
& & Timescales (s) & \\
$\frac{1}{2} v_{t h}$ & $3.21 \times 10^{-8}$ & $9.10 \times 10^{-8}$ & $1.67 \times 10^{-7}$ \\
$v_{\text {th }}$ & $4.87 \times 10^{-8}$ & $1.38 \times 10^{-7}$ & $2.53 \times 10^{-7}$ \\
$2 v_{t h}$ & $1.75 \times 10^{-7}$ & $4.94 \times 10^{-7}$ & $9.05 \times 10^{-7}$ \\
$4 v_{t h}$ & $1.34 \times 10^{-6}$ & $3.77 \times 10^{-6}$ & $6.95 \times 10^{-6}$ \\
\hline \hline
\end{tabular}


TABLE II. Mean-free-paths and timescales for electron-electron collisions, at a range of electron temperatures and multiples of the electron thermal speed, for a hole density of $10^{9} \mathrm{~cm}^{-3}$.

\begin{tabular}{lccc}
\hline \hline$T_{e}=$ & $25 \mathrm{~K}$ & $48 \mathrm{~K}$ & $105 \mathrm{~K}$ \\
\hline & & Mean-free-paths (cm) & \\
$\frac{1}{2} v_{t h}$ & $6.30 \times 10^{-3}$ & $1.89 \times 10^{-2}$ & $7.40 \times 10^{-2}$ \\
$v_{\text {th }}$ & $1.91 \times 10^{-2}$ & $5.75 \times 10^{-2}$ & $2.26 \times 10^{-1}$ \\
$2 v_{t h}$ & $1.37 \times 10^{-1}$ & $4.12 \times 10^{-1}$ & $1.62 \times 10^{0}$ \\
$4 v_{\text {th }}$ & $2.09 \times 10^{0}$ & $6.30 \times 10^{0}$ & $2.47 \times 10^{1}$ \\
& & Timescales (s) & \\
$\frac{1}{2} v_{t h}$ & $4.56 \times 10^{-9}$ & $9.90 \times 10^{-9}$ & $2.63 \times 10^{-8}$ \\
$v_{\text {th }}$ & $6.90 \times 10^{-9}$ & $1.51 \times 10^{-8}$ & $4.00 \times 10^{-8}$ \\
$2 v_{\text {th }}$ & $2.48 \times 10^{-8}$ & $5.40 \times 10^{-8}$ & $1.43 \times 10^{-7}$ \\
$4 v_{\text {th }}$ & $1.90 \times 10^{-7}$ & $4.12 \times 10^{-7}$ & $1.10 \times 10^{-6}$ \\
\hline \hline
\end{tabular}

\section{B. Self-similar plasma expansion and ion hole propagation}

The inhomogeneous density distribution and overall plasma expansion introduce additional complications not described by the simple wave equation (Eq. (6)). However, with a proper treatment of these effects, it is possible to measure the ion-hole speed and check the ion-acoustic-wave description. For a spherically symmetric Gaussian density, under the assumptions of quasi-neutrality and adiabaticity, the evolution of the size of the plasma cloud $(1 / \sqrt{e}$ density radius, $\sigma$ ), the expansion velocity (hydrodynamic velocity, $\mathbf{u})$, and the ion and electron temperatures $\left(T_{i}\right.$ and $\left.T_{e}\right)$ can be written as ${ }^{17}$

$$
\begin{gathered}
\sigma^{2}(t)=\sigma^{2}(0)\left(1+\frac{t^{2}}{\tau_{\exp }^{2}}\right), \\
\gamma(t)=\frac{t / \tau_{\exp }^{2}}{1+t^{2} / \tau_{\exp }^{2}}, \\
\mathbf{u}(\mathbf{r}, t)=\gamma(t) \mathbf{r} \\
T_{\alpha}(t)=\frac{T_{\alpha}(0)}{1+t^{2} / \tau_{\exp }^{2}},
\end{gathered}
$$

where $\mathbf{r}$ is the radial distance from cloud center and the expansion rate is determined by the characteristic expansion time

$$
\tau_{\exp }=\sqrt{\frac{m_{\mathrm{i}} \sigma(0)^{2}}{k_{\mathrm{B}}\left[T_{e}(0)+T_{i}(0)\right]}} .
$$

For a strontium plasma with a typical initial size of $\sigma(0)$ $=1.4 \mathrm{~mm}$, an initial electron temperature, $T_{e}(0)=25(55) \mathrm{K}$, and an initial ion temperature, $T_{i}(0)=1 \mathrm{~K}, \tau_{\exp }$ is approximately equal to $29(20) \mu \mathrm{s}$.

The plasma expands self-similarly, maintaining a Gaussian profile (Eq. (7)). From Eqs. (8) and (9), one can see that the expansion velocity is proportional to position from the center and initially proportional to time for $t<\tau_{\exp }$. For $t \gg \tau_{\exp }, \gamma(t) \approx 1 / t$, and acceleration decreases. Finally, Eq. (10) shows adiabatic cooling of the temperature of the ions and electrons.
Intuition for the evolution of the gap can be gained by recognizing that both the plasma expansion and the initial evolution of the gap are driven by the conversion of electron thermal energy into directed ion motion. This process can be understood hydrodynamically using the coupled ion-electron momentum balance equations at early times when ion velocities are small. ${ }^{16}$ The initial acceleration of the ions $\left(a_{0}\right)$ is provided by the electron pressure $\left(P_{e}\right)$ according to $M n a_{0}$ $\approx-\nabla P_{e} \approx-\nabla\left(n k_{B} T_{e}(0)\right) . T_{e}(0)$ is expected to be close to uniform, ${ }^{25}$ and the final ion velocity is set by conservation of energy, $v_{f} \approx \sqrt{k_{B} T_{e}(0) / M}$. This implies a timescale of

$$
\tau \approx \frac{v_{f}}{a_{0}} \approx-\frac{n}{\nabla n} \sqrt{\frac{M}{k_{B} T_{e}(0)}} .
$$

For the expansion, the length scale is set by the Gaussian density profile, $-n / \nabla n \approx \sigma_{0}$, yielding a selfsimilar expansion with the characteristic expansion time of $\tau_{\exp } \approx{\sqrt{M \sigma_{0}^{2} /\left[k_{B} T_{e}(0)\right]}}^{2,17}$ an approximation of Eq. (11).

The presence of a density depletion introduces a smaller length scale, $-n / \nabla n \approx \ell$, leading to a faster timescale for density change associated with ions moving into the gap, $\tau_{\text {fast }} \approx \sqrt{M \ell^{2} /\left[k_{B} T_{e}(0)\right]}$. This yields $\tau_{\text {fast }}=5 \mu$ s for $T_{e}(0)$ $=25 \mathrm{~K}$, which approximately matches the experimental timescale for the filling of the gap and emergence of two ion holes.

To check the overall expansion and the electron temperature, we fit the evolution of the plasma size obtained from Gaussian fits to the density distributions to the expected evolution for self similar expansion, Eq. (7). Figure 3 shows these measurements and fits. The extracted temperatures from the size evolution are in reasonable agreement with the expected self-similar expansion of an unperturbed plasma cloud, demonstrating the gap is a small perturbation that does not significantly modify the expansion in this regime.

To model the motion of the ion holes, we assume propagation at the ion acoustic sound speed $C_{s}\left(T_{e}\right)$ in the frame of reference moving with the expanding background plasma, $u_{x}=\gamma(t) x$ (Eq. (9)), allowing the initial electron temperature to vary when fitting the data. This description is reasonable, as suggested above, because an ion hole can be viewed as an ion acoustic wavepacket comprised of a linear superposition of waves with wavelengths $\sim \ell$. This length scale is much larger than the Debye screening length $\left(\lambda_{D}=10 \mu \mathrm{m}\right.$ for

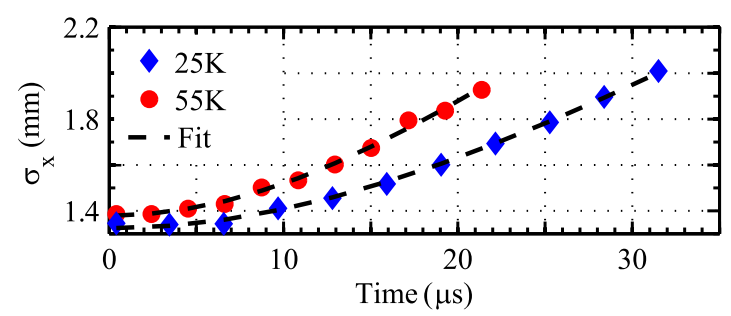

FIG. 3. Evolution of the plasma size for initial peak density $n_{0} \sim 1$ $\times 10^{15} \mathrm{~m}^{-3}$ and initial electron temperature $T_{e}(0)$ determined by the photoionization laser indicated in the legend. The plasma size was measured from the Gaussian fits neglecting the small perturbation of the ion holes (see Fig. 2) and fit to Eq. (7). The extracted values of $T_{e}(0)$ from expansion are $43 \pm 3 \mathrm{~K}$ and $24 \pm 4 \mathrm{~K}$. 
$T_{e}=25 \mathrm{~K}$ ), so these waves are dispersionless with speed $C_{s}$. For the small hole amplitudes used, we neglect nonlinearities and assume the background density and electron temperature evolve according to the self similar Gaussian expansion of an unperturbed plasma ${ }^{17}$ (Eqs. (7)-(11)). This leads to the following differential equation for the evolution of the ion hole position

$$
\begin{aligned}
\dot{x} & =\gamma(t) x+C_{s}(t) \\
& =\frac{t / \tau_{\exp }^{2}}{1+t^{2} / \tau_{\exp }^{2}} x+\sqrt{\frac{k_{B}}{m} \frac{T_{e}(0)}{1+t^{2} / \tau_{\exp }^{2}}},
\end{aligned}
$$

where $\dot{x}$ is the derivative with respect to time and $x=0$ corresponds to the center of the plasma. Equation (13) has an analytic solution as follows:

$$
\begin{aligned}
x & = \pm\left[x_{0}+\sigma_{0} \arctan \left(\frac{t}{\tau_{\exp }}\right)\right] \sqrt{1+t^{2} / \tau_{\exp }^{2}}, \\
& \approx \pm x_{0}\left(1+\frac{t^{2}}{2 \tau_{\exp }^{2}}\right) \pm \sqrt{\frac{k_{B} T_{e}(0)}{m}} t\left(1+\frac{t^{2}}{6 \tau_{\exp }^{2}}\right) .
\end{aligned}
$$

The second line shows that the holes move linearly for $t \ll \tau_{\exp }$. In the limit $t \gg \tau_{\exp }$, we also find linear propagation at a slightly higher speed,

$$
x \approx \pm \frac{x_{0} t}{\tau_{\exp }} \pm \frac{\pi}{2} \sqrt{\frac{k_{B} T_{e}(0)}{m}} t
$$

and our measurements do reach into the crossover regime between the two limits.

To quantitatively analyze propagation of the ion holes, we first measure the holes' locations and widths by fitting each density profile to the sum of three Gaussians, i.e., the overall cloud and two holes. At early times, when the gap has widened but not yet split, we find the hole centers by making the simplifying assumption that the widths of each hole are unchanged from the width of the initial gap. Holes are known to interact attractively in the kinetic regime, ${ }^{48}$ so the evolution of the gap at early times is an important topic for further study.

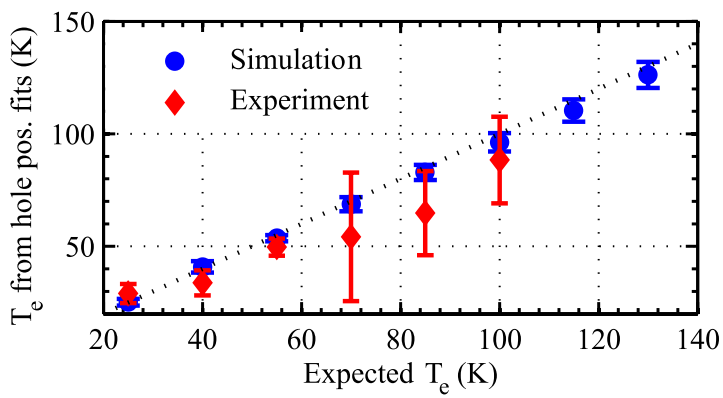

FIG. 4. Measured $T_{e}(0)$ extracted from the motion of the ion holes (Eq. (14)). The expected electron temperature is the value set by the wavelength of the photoionizing laser. Simulation and experimental points are both extracted the same way from the density profiles, and each point is an average of the measurement for left and right-going holes. The dashed line is provided as a guide to the eye. Error bars here and elsewhere in the paper are $95 \%$ confidence intervals.
The evolution of the positions of the holes is then fit to Eq. (14), with $x_{0}$ and $\tau_{\text {exp }}$ as the fitting parameters and $\sigma_{0}$ is obtained from 2D Gaussian fits to the plasma's areal density, shown in Figure 2(c). Figure 4 shows that the extracted initial electron temperatures match the expected values from the photoionization laser detuning, supporting the model for hole propagation in this regime (Eq. (14)). Alternatively, this can be viewed as an extraction of the ion hole velocity and confirmation that it closely matches the local ion sound speed. We also analyze the simulation results using the same procedure and show the extracted initial electron temperatures in Fig. 4, which agree well with expectations and data.

\section{LIF spectroscopy and studying the ion hole through velocity}

A complementary picture of the ion hole dynamics and important additional information on the ion temperature and possible kinetic effects can be obtained by looking at the LIF spectra of small spatial regions in the vicinity of the gap. As described in Sec. II, these can be interpreted as velocity distribution functions because of the sensitivity of the spectrum to Doppler effects. Figure 5 shows spectra of the central region and regions to the right of the gap for small amplitude initial density perturbations. Due to the symmetry of our plasma about the plane of the initial density depletion, corresponding regional spectra in the two hemispheres mirror each other.

A positive average velocity represents ions moving away from plasma center, whereas, negative velocity represents ions moving towards it. Without an initial density perturbation, regional spectra show average positive velocities that increase with time and distance from center as expected for a self-similar plasma expansion. ${ }^{17}$ The widths reflect the

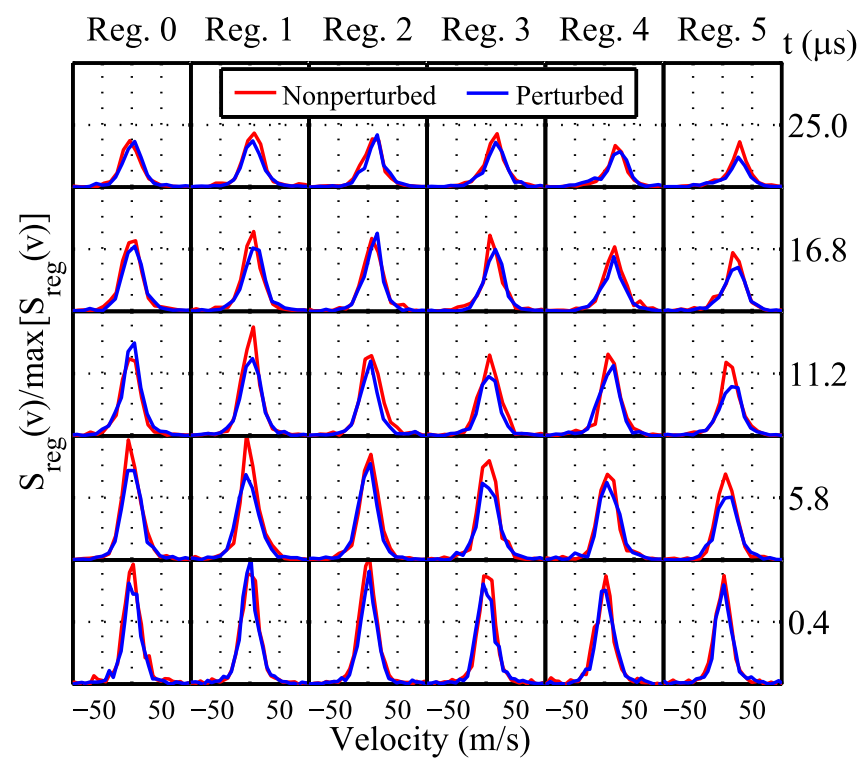

FIG. 5. Spatially resolved regional spectra for an unperturbed (blue) and perturbed (red) plasma with $T_{e}(0)=40 \mathrm{~K}, \sigma_{0}=1.4 \mathrm{~mm}$, and $n_{0} \sim 1 \times 10^{9} \mathrm{~cm}^{-3}$. Each region is $\sigma(t) / 10$ in length perpendicular and parallel to the gap. Region 0 represents the center of the plasma, and regions are spaced every $\sigma(t) / 4$ $\sim 350 \mu \mathrm{m}$ to the right of center with respect to the orientation of Fig. 1 . Time since ionization is indicated on the right. The amplitudes of all signals are scaled by a universal factor. 
ion temperature set by disorder-induced heating. ${ }^{3,7}$ It is very difficult to discern by eye a difference between the spectra with and without an initial density perturbation. This lack of difference shows that the ion velocity distribution is staying Maxwellian in the presence of the ion hole, as expected for hydrodynamic conditions. Kinetic conditions for the ions would likely result in skewed and flat-top distributions, or, in the extreme, bimodal distributions and evidence for multiple streaming populations of ions.

For finer analysis, spectra can be fit to Voigt profiles to extract ion velocity and temperature. Figure 6 shows such an analysis for spectra of the central region and regions to the right of the gap. For perturbed plasmas, as the hole moves into a region, the density gradient at the leading edge accelerates ions towards the center of the plasma. This counter flow of the hole velocity and underlying fluid velocity of the medium is the characteristic of hydrodynamic hole phenomena. For $350 \mu \mathrm{m}$ from plasma center (region 1, Fig. 6 (bottom)), counterflow is evident within a few $\mu$ s after plasma creation as negative velocity, indicating ions moving towards plasma center. The gradient at the trailing edge of the hole subsequently decelerates the ions and creates a stagnation point so that after passage of the hole, the ion velocity returns to the increasing, positive value indicative of overall plasma expansion. Note that higher initial electron temperature produces larger negative fluid velocity and causes the ion hole to move more quickly through the regions. In regions further from the plasma center, the ion hole arrives later, and the deviation in velocity associated with the passage of the ion hole is smaller compared to the expansion velocity. For $1050 \mu \mathrm{m}$ from plasma center, for example (region 3 ), the overall ion velocity is never negative, but in the frame of reference expanding with the plasma, the perturbation still represents a movement of ions back towards the initial gap

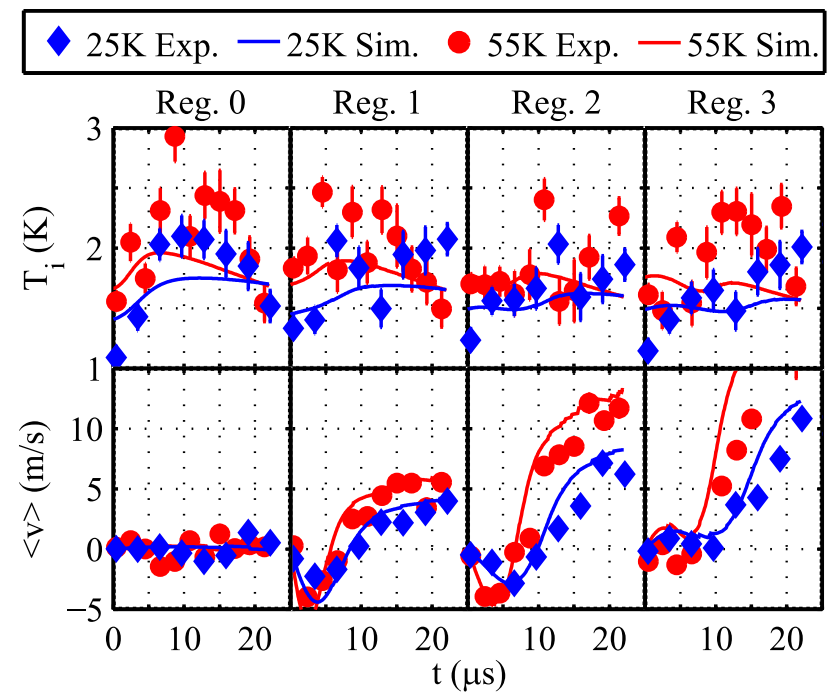

FIG. 6. Average ion velocity and temperature for regions in the vicinity of the gap. Each region is $\sigma(t) / 10 \sim 140 \mu \mathrm{m}$ perpendicular to the gap and the same parallel to it. Region 0 represents the center of the plasma, and subsequent regions are each shifted to the right by $\sigma(t) / 4 \sim 350 \mu \mathrm{m}$ with respect to the orientation of Fig. 2. Results of the simulation are also shown. Note the presence of negative velocities, which indicate ion flow towards the center as the ion hole passes as it moves away from the center. as the ion hole passes. The hydrodynamic simulation reproduces the data well.

The sound speed at $T_{e}=25(55) \mathrm{K}$ is approximately $48(72) \mathrm{m} / \mathrm{s}$, and Fig. 6 shows that the bulk flows are subsonic, with flow speeds generally lower than $10 \mathrm{~m} / \mathrm{s}$. A stronger density gradient in the initial conditions should induce a stronger pressure gradient and drive faster flows. This may make it possible to enter the super-sonic regime, and thereby study the formation and interaction of shocks. ${ }^{54,55} \mathrm{We}$ plan to pursue this in a future investigation.

In a system that is well described as hydrodynamic, the passing of an ion hole represents an adiabatic rarefaction followed by recompression, which leads to localized cooling and then reheating of the ions. This effect is small, but visible in the hydrodynamic simulation (Fig. 6). It is not possible to discern this against the noise in the experimental data.

\section{CONCLUSION}

In conclusion, we have observed ion holes in an ultracold neutral plasma in both position and velocity space, and we have confirmed that they propagate at the local sound speed. These holes are formed by splitting of an initially stationary density perturbation, and they are well described by a one-dimensional, two-fluid, hydrodynamic simulation. These studies demonstrate the potential of using UNPs and numerical simulations to study a wide range of fundamental plasma phenomena. There are many future directions to explore with these capabilities. For example, sharper density features may lead to the excitation of shocks. ${ }^{54,55}$ Plasmas with initial localized density enhancements should produce solitons. ${ }^{37,38}$ Because of the ability to measure the ion velocity distribution, we can study the crossover from the hydrodynamic to kinetic regime, such as by varying the density of ions and length scale of the initial gap. We can look for the onset of streaming plasmas, which may also enable a measurement of the ion stopping power. ${ }^{56}$ This is particularly interesting because the ions, at least initially, are strongly coupled. ${ }^{2,50}$

\section{ACKNOWLEDGMENTS}

This work was supported by the Department of Energy and National Science Foundation (PHY-0714603) and the Air Force Office of Scientific Research (FA9550-12-10267).

${ }^{1}$ T. C. Killian, S. Kulin, S. D. Bergeson, L. A. Orozco, C. Orzel, and S. L. Rolston, Phys. Rev. Lett. 83, 4776 (1999).

${ }^{2}$ T. C. Killian, T. Pattard, T. Pohl, and J. M. Rost, Phys. Rep. 449, 77 (2007).

${ }^{3}$ C. E. Simien, Y. C. Chen, P. Gupta, S. Laha, Y. N. Martinez, P. G. Mickelson, S. B. Nagel, and T. C. Killian, Phys. Rev. Lett. 92, 143001 (2004).

${ }^{4}$ Y. C. Chen, C. E. Simien, S. Laha, P. Gupta, Y. N. Martinez, P. G. Mickelson, S. B. Nagel, and T. C. Killian, Phys. Rev. Lett. 93, 265003 (2004).

${ }^{5}$ E. A. Cummings, J. E. Daily, D. S. Durfee, and S. D. Bergeson, Phys. Rev. Lett. 95, 235001 (2005).

${ }^{6}$ G. Bannasch, J. Castro, P. McQuillen, T. Pohl, and T. C. Killian, Phys. Rev. Lett. 109, 185008 (2012).

${ }^{7}$ M. S. Murillo, Phys. Rev. Lett. 87, 115003 (2001).

${ }^{8}$ M. S. Murillo, Phys. Rev. Lett. 96, 165001 (2006). 
${ }^{9}$ T. Pohl, T. Pattard, and J. M. Rost, Phys. Rev. Lett. 92, 155003 (2004).

${ }^{10}$ T. Pohl, T. Pattard, and J. M. Rost, Phys. Rev. A 70, 033416 (2004).

${ }^{11}$ M. Rosenberg and P. K. Shukla, Phys. Scr. 83, 015503 (2011).

${ }^{12}$ M. Lyon, S. D. Bergeson, and M. S. Murillo, Phys. Rev. E 87, 033101 (2013).

${ }^{13}$ S. Mazevet, L. A. Collins, and J. D. Kress, Phys. Rev. Lett. 88, 55001 (2002).

${ }^{14}$ T. Pohl, T. Pattard, and J. M. Rost, Phys. Rev. Lett. 94, 205003 (2005).

${ }^{15}$ X. L. Zhang, R. S. Fletcher, S. L. Rolston, P. N. Guzdar, and M. Swisdak, Phys. Rev. Lett. 100, 235002 (2008).

${ }^{16}$ S. Kulin, T. C. Killian, S. D. Bergeson, and S. L. Rolston, Phys. Rev. Lett. 85, 318 (2000).

${ }^{17}$ S. Laha, P. Gupta, C. E. Simien, H. Gao, J. Castro, and T. C. Killian, Phys. Rev. Lett. 99, 155001 (2007).

${ }^{18}$ K. A. Twedt and S. L. Rolston, Phys. Rev. Lett. 108, 065003 (2012).

${ }^{19}$ R. S. Fletcher, X. L. Zhang, and S. L. Rolston, Phys. Rev. Lett. 96, 105003 (2006).

${ }^{20}$ A. Lyubonko, T. Pohl, and J.-M. Rost, New J. Phys. 14, 053039 (2012).

${ }^{21}$ J. Castro, P. McQuillen, and T. C. Killian, Phys. Rev. Lett. 105, 065004 (2010).

${ }^{22}$ X. L. Zhang, R. S. Fletcher, and S. L. Rolston, Phys. Rev. Lett. 101, 195002 (2008).

${ }^{23}$ T. C. Killian, M. J. Lim, S. Kulin, R. Dumke, S. D. Bergeson, and S. L. Rolston, Phys. Rev. Lett. 86, 3759 (2001).

${ }^{24}$ F. Robicheaux and J. D. Hanson, Phys. Rev. Lett. 88, 55002 (2002).

${ }^{25}$ F. Robicheaux and J. D. Hanson, Phys. Plasmas 10, 2217 (2003).

${ }^{26}$ S. G. Kuzmin and T. M. O'Neil, Phys. Plasmas 9, 3743 (2002).

${ }^{27}$ S. G. Kuzmin and T. M. O'Neil, Phys. Rev. Lett. 88, 65003 (2002).

${ }^{28}$ P. Gupta, S. Laha, C. E. Simien, H. Gao, J. Castro, T. C. Killian, and T. Pohl, Phys. Rev. Lett. 99, 75005 (2007).

${ }^{29}$ R. S. Fletcher, X. L. Zhang, and S. L. Rolston, Phys. Rev. Lett. 99, 145001 (2007).

${ }^{30}$ M. P. Robinson, B. L. Tolra, M. W. Noel, T. F. Gallagher, and P. Pillet, Phys. Rev. Lett. 85, 4466 (2000).

${ }^{31}$ T. Pohl, T. Pattard, and J. M. Rost, Phys. Rev. A 68, 010703 (2003).
${ }^{32}$ T. F. Gallagher, P. Pillet, M. P. Robinson, B. Laburthe-Tolra, and M. W. Noel, J. Opt. Soc. Am. B 20, 1091 (2003).

${ }^{33}$ P. McQuillen, X. Zhang, T. Strickler, F. Dunning, and T. Killian, Phys. Rev. A 87, 013407 (2013).

${ }^{34}$ J. P. Morrison, C. J. Rennick, J. S. Keller, and E. R. Grant, Phys. Rev. Lett. 101, 205005 (2008).

${ }^{35}$ B. Eliasson and P. Shukla, Phys. Rep. 422, 225 (2006).

${ }^{36}$ H. Schamel, Phys. Plasmas 16, 113709 (2009).

${ }^{37}$ J. D. Moody and C. F. Driscoll, Phys. Plasmas 2, 4482 (1995).

${ }^{38}$ M. Q. Tran, Phys. Scr. 20, 317 (1979).

${ }^{39}$ H. Schamel and S. Bujarbarua, Phys. Fluids 23, 2498 (1980).

${ }^{40}$ H. L. Pecseli, R. J. Armstrong, and J. Trulsen, Phys. Lett. 81, 386 (1981).

${ }^{41}$ S. J. Bradshaw and H. E. Mason, Astron. Astrophys. 407, 1127 (2003).

${ }^{42}$ S. J. Bradshaw and J. A. Klimchuk, Astrophys. J. Suppl. Ser. 194, 26 (2011).

${ }^{43}$ P. K. Shukla, Phys. Plasmas 7, 1044 (2000).

${ }^{44}$ M. R. Andrews, D. M. Kurn, H.-J. Miesner, D. S. Durfee, C. G.

Townsend, S. Inouye, and W. Ketterle, Phys. Rev. Lett. 79, 553 (1997).

${ }^{45}$ J. Joseph, B. Clancy, L. Luo, J. Kinast, A. Turlapov, and J. Thomas, Phys. Rev. Lett. 98, 170401 (2007)

${ }^{46}$ D. Jovanović and P. K. Shukla, Phys. Rev. Lett. 90, 135001 (2003).

${ }^{47}$ B. Eliasson and P. Shukla, Phys. Rev. Lett. 92, 095006 (2004).

${ }^{48}$ K. Saeki, P. Michelson, H. L. Pecseli, and J. J. Rasmussen, Phys. Rev. Lett. 42, 501 (1979).

${ }^{49}$ H. Schamel, Phys. Plasmas 19, 020501 (2012).

${ }^{50}$ S. Ichimaru, Rev. Mod. Phys. 54, 1017 (1982).

${ }^{51}$ K. R. Castleman, Digital Image Processing (Pearson Education, 2007).

${ }^{52}$ J. Castro, H. Gao, and T. C. Killian, Plasma Phys. Controlled Fusion 50, 124011 (2008).

${ }^{53}$ T. H. Stix, Waves in Plasmas, 2nd ed. (AIP, New York, 1992).

${ }^{54}$ Y. Nakamura, H. Bailung, and P. K. Shukla, Phys. Rev. Lett. 83, 1602 (1999).

${ }^{55}$ H. Ikezi, R. Taylor, and D. Baker, Phys. Rev. Lett. 25, 11 (1970).

${ }^{56}$ D. O. Gericke, M. Schlanges, and Th. Bornath, Phys. Rev. E 65, 036406 (2002). 